\title{
ANÁlisis DEL CONSUMO Y DE LA IMAGEN DE LA CARNE DE POLLO Y PAVO EN LOS HOGARES CON NIÑOS EN ESPAÑA
}

\author{
Amparo Baviera-Puig ${ }^{a *}, \underline{\text { Carmen Escribá-Pérez }}{ }^{a}$, Luis Montero-Vicente ${ }^{a}$ y Juan Buitrago-Vera ${ }^{a}$ \\ ${ }^{a}$ Universitat Politècnica de València (Valencia, ambapui@upv.es).
}

\begin{abstract}
Resumen
Estudios previos muestran que, comercialmente, la carne de pollo tiene un posicionamiento similar al de la carne de pavo. Así, en la mente de los consumidores ambas son percibidas como carnes saludables y bajas en grasa. Debido a estas características, su consumo es ideal para los niños. Sin embargo, apenas existen investigaciones sobre el consumo y la imagen de ambas carnes en las familias con niños. El objetivo es doble: i) analizar el consumo de carne de pollo y pavo en hogares con niños (menores de 18 años); ii) analizar la imagen de la carne de pollo y pavo en los hogares con niños para comprobar si existen diferencias.

Para ello, realizamos una encuesta telefónica con un error de $\pm 4,0 \%$ a un nivel de confianza del 95,5\% en España. Obtenemos que la carne de pavo fresca y los elaborados de pollo y pavo se compran con más frecuencia en los hogares con niños. Si analizamos la imagen de ambas carnes en los hogares con niños con respecto a varios ítems, en todos los ítems la valoración del pollo es mayor excepto en "Es una carne sana y saludable", "Es una carne de alta calidad" y "Es digestiva, no pesada".
\end{abstract}

Palabras clave: hábitos de compra, hábitos de consumo, frecuencia de compra, imagen.

\section{Introducción}

La carne es una fuente importante de proteínas, aminoácidos esenciales, vitaminas del grupo B, minerales y otros compuestos bioactivos (Martini et al., 2019). El consumo de carne es muy recomendable, ya que favorece el desarrollo de las funciones corporales y cognitivas en niños y adolescentes, así como en bebés (Cofnas, 2019; Tang et al., 2018). Específicamente, el pollo y el pavo se recomiendan por sus propiedades nutricionales y dietéticas, ya que son más ligeros que otras carnes (Pereira y Vicente, 2013).

Estudios previos muestran que, comercialmente, la carne de pollo tiene un posicionamiento similar al de la carne de pavo (Baviera-Puig et al., 2021; Montero-Vicente et al., 2018). En la mente de los consumidores, ambos tipos son percibidos como carnes saludables y bajas en grasa. Debido a estas características, su consumo es ideal para los niños. Sin embargo, apenas existen investigaciones sobre el consumo y la imagen de ambas carnes en las familias con niños.

El objetivo del presente artículo es doble: i) analizar los hábitos de consumo y compra de las carnes de pollo y pavo por parte de los hogares con niños, ya que ambos tipos de carne son más saludables que otros; y ii) analizar la imagen de la carne de pollo y pavo en el total de la muestra y en los hogares con niños para comprobar si existen diferencias entre ellos.

\section{Metodología}

Se realizó una encuesta telefónica en España peninsular, es decir, en toda la península, excluyendo Baleares, Canarias, Ceuta y Melilla. La entrevista fue telefónica y se realizó mediante un sistema CATI (Computer Assisted Telephone Interview), es decir, asistida por ordenador, y un cuestionario estructurado. Los números de teléfono se seleccionaron al azar de las guías telefónicas públicas. Las entrevistas duraron aproximadamente 12 minutos. No hubo ningún tipo de compensación para los entrevistados.

El tamaño de la muestra fue de 625 entrevistas, para un error de $\pm 4,0 \%$ y un nivel de confianza del 95,5\%. El error fue inferior al límite deseable del 4\% indicado por Cea (2010) en la investigación social. El perfil del consumidor seleccionado es el de la persona que se encarga de la compra de alimentos o comparte esta responsabilidad en los hogares donde se consume carne de pollo y pavo, aunque sea de forma esporádica. La edad de los entrevistados osciló entre los 18 y los 75 años.

Una vez conseguidos los datos, calculamos tabulaciones cruzadas para diferenciar los distintos tipos de hogares analizados. Comparamos el total de la muestra y los hogares con y sin hijos menores de 18 años. También distinguimos entre carne fresca y elaborada. Se utilizó el test de la ji cuadrado como prueba estadística de significación. La escala utilizada para medir la imagen es propuesta por los propios autores a partir de las escalas utilizadas en otros trabajos, tales como, Montero-Vicente et al. (2018). 


\section{Resultados}

\subsection{Análisis de la frecuencia de consumo y de compra}

Al analizar la frecuencia de consumo, sólo encontramos diferencias significativas entre los hogares con y sin hijos menores de 18 años para la carne de pavo elaborada (Cuadro 1).

Cuadro 1. Consumo de elaborados de pavo en función de si viven menores de 18 años o no

\begin{tabular}{|c|c|c|c|c|c|c|}
\hline \multirow{3}{*}{$\begin{array}{l}\text { Frecuencia de } \\
\text { consumo }\end{array}$} & \multirow{2}{*}{\multicolumn{2}{|c|}{ Total }} & \multicolumn{4}{|c|}{ Viven menores de 18 años en el hogar } \\
\hline & & & \multicolumn{2}{|c|}{$\mathrm{Si}$} & \multicolumn{2}{|c|}{ No } \\
\hline & Frecuencia & $\%$ & Frecuencia & $\%$ & Frecuencia & $\%$ \\
\hline $\begin{array}{l}\text { Una vez a la semana } \\
\text { o más } \\
\text { frecuentemente }\end{array}$ & 246 & 58,85 & 71 & 49,65 & 175 & 63,64 \\
\hline Cada dos semanas & 75 & 17,94 & 34 & 23,78 & 41 & 14,91 \\
\hline Una vez al mes & 53 & 12,68 & 24 & 16,78 & 29 & 10,55 \\
\hline $\begin{array}{l}\text { Una vez cada dos } \\
\text { meses }\end{array}$ & 24 & 5,74 & 6 & 4,20 & 18 & 6,55 \\
\hline $\begin{array}{l}\text { Menos de una vez } \\
\text { cada dos meses }\end{array}$ & 14 & 3,35 & 7 & 4,90 & 7 & 2,55 \\
\hline Nunca & 6 & 1,44 & 1 & 0,70 & 5 & 1,82 \\
\hline Total & 418 & 100,00 & 143 & 100,00 & 275 & 100,00 \\
\hline
\end{tabular}

Fuente: Elaboración propia

Al analizar la frecuencia de compra, obtenemos diferencias significativas en función de si viven menores de 18 años o no para la carne fresca de pavo (Cuadro 2), los elaborados de pavo (Cuadro 3) y los elaborados de pollo (Cuadro 4). En los 3 casos, se compran con más frecuencia en los hogares donde hay menores.

Cuadro 2. Frecuencia de compra de carne fresca de pavo en función de si viven menores de 18 años o no

\begin{tabular}{|l|c|c|c|c|c|c|}
\hline \multirow{2}{*}{$\begin{array}{l}\text { Frecuencia de } \\
\text { compra }\end{array}$} & \multicolumn{3}{|c|}{ Total } & \multicolumn{3}{c|}{ Viven menores de 18 años en el hogar } \\
\cline { 2 - 7 } & Frecuencia & $\%$ & Frecuencia & $\%$ & Frecuencia & $\%$ \\
\hline $\begin{array}{l}\text { Una vez a la semana } \\
\text { o más } \\
\text { frecuentemente }\end{array}$ & 234 & 37,44 & 82 & 44,32 & 152 & 34,55 \\
\hline Cada dos semanas & 90 & 14,40 & 25 & 13,51 & 65 & 14,77 \\
\hline Una vez al mes & 75 & 12,00 & 28 & 15,14 & 47 & 10,68 \\
\hline $\begin{array}{l}\text { Una vez cada dos } \\
\text { meses }\end{array}$ & 35 & 5,60 & 9 & 4,86 & 26 & 5,91 \\
\hline $\begin{array}{l}\text { Menos de una vez } \\
\text { cada dos meses }\end{array}$ & 38 & 6,08 & 9 & 4,86 & 29 & 6,59 \\
\hline Nunca & 153 & 24,48 & 32 & 17,30 & 121 & 27,50 \\
\hline Total & 625 & 100,00 & 185 & 100,00 & 440 & 100,00 \\
\hline
\end{tabular}

Ji cuadrado con 5 grados de libertad $=12,0522 \quad(\mathrm{p}=0,0341)$

Fuente: Elaboración propia 
Cuadro 3. Frecuencia de compra de elaborados de pavo en función de si viven menores de 18 años o no

\begin{tabular}{|l|c|c|c|c|c|c|}
\hline \multirow{2}{*}{$\begin{array}{l}\text { Frecuencia de } \\
\text { compra }\end{array}$} & \multicolumn{3}{|c|}{ Total } & \multicolumn{3}{c|}{ Viven menores de 18 años en el hogar } \\
\cline { 4 - 7 } & Frecuencia & $\%$ & \multicolumn{2}{c|}{ Si } & \multicolumn{2}{c|}{ No } \\
\hline $\begin{array}{l}\text { Una vez a la semana } \\
\text { o más } \\
\text { frecuentemente }\end{array}$ & 207 & 33,12 & 69 & 37,30 & 138 & 31,36 \\
\hline Cada dos semanas & 93 & 14,88 & 30 & 16,22 & 63 & 14,32 \\
\hline Una vez al mes & 76 & 12,16 & 33 & 17,84 & 43 & 9,77 \\
\hline $\begin{array}{l}\text { Una vez cada dos } \\
\text { meses }\end{array}$ & 21 & 3,36 & 5 & 2,70 & 16 & 3,64 \\
\hline $\begin{array}{l}\text { Menos de una vez } \\
\text { cada dos meses }\end{array}$ & 22 & 3,52 & 7 & 3,78 & 15 & 3,41 \\
\hline Nunca & 206 & 32,96 & 41 & 22,16 & 165 & 37,50 \\
\hline Total & 625 & 100,00 & 185 & 100,00 & 440 & 100,00 \\
\hline
\end{tabular}

Ji cuadrado con 5 grados de libertad $=18,3522 \quad(\mathrm{p}=0,0025)$

Fuente: Elaboración propia

Cuadro 4. Frecuencia de compra de elaborados de pollo en función de si viven menores de 18 años o no

\begin{tabular}{|l|c|c|c|c|c|c|}
\hline \multirow{2}{*}{$\begin{array}{l}\text { Frecuencia de } \\
\text { compra }\end{array}$} & \multicolumn{3}{|c|}{ Total } & \multicolumn{3}{c|}{ Viven menores de 18 años en el hogar } \\
\cline { 2 - 7 } & Frecuencia & $\%$ & Frecuencia & $\%$ & Frecuencia & $\%$ \\
\hline $\begin{array}{l}\text { Una vez a la semana } \\
\text { o más } \\
\text { frecuentemente }\end{array}$ & 177 & 28,32 & 68 & 36,76 & 109 & 24,77 \\
\hline Cada dos semanas & 87 & 13,92 & 37 & 20,00 & 50 & 11,36 \\
\hline Una vez al mes & 74 & 11,84 & 27 & 14,59 & 47 & 10,68 \\
\hline $\begin{array}{l}\text { Una vez cada dos } \\
\text { meses }\end{array}$ & 19 & 3,04 & 8 & 4,32 & 11 & 2,50 \\
\hline $\begin{array}{l}\text { Menos de una vez } \\
\text { cada dos meses }\end{array}$ & 20 & 3,20 & 7 & 3,78 & 13 & 2,95 \\
\hline Nunca & 248 & 39,68 & 38 & 20,54 & 210 & 47,73 \\
\hline \multicolumn{2}{|c|}{625} & 100,00 & 185 & 100,00 & 440 & 100,00 \\
\hline
\end{tabular}

Fuente: Elaboración propia

\subsection{Análisis de la imagen}

Si analizamos la imagen de ambas carnes en el total de la muestra y en los hogares con menores de 18 años respecto a varios ítems, obtenemos resultados similares (Cuadro 5). En todos los ítems, la valoración del pollo es superior, excepto en "Es una carne sana y saludable" y "Es una carne de alta calidad".

La única diferencia entre ambos grupos es el ítem "Es digestiva, no es pesada". En el total de la muestra, el pollo $(83,84 \%)$ tiene una mayor valoración que la carne de pavo $(81,44 \%)$, mientras que en los hogares con menores de 18 años el pavo $(87,03 \%)$ obtiene una mayor valoración que la carne de pollo $(83,24 \%)$. Aunque el patrón es diferente, los porcentajes son muy similares.

El pollo destaca en los siguientes ítems: "Se encuentra fácilmente en los establecimientos donde realizo las compras ", "Tiene una buena relación calidad-precio", "Es una carne sabrosa", "Es una carne fácil y rápida de cocinar", "Se puede preparar de muchas maneras" y "Es una carne con un precio atractivo, económico". 
Cuadro 5. Imagen de la carne de pollo y pavo (\%)

\begin{tabular}{|l|c|c|c|c|}
\hline \multirow{2}{*}{ Variable } & \multicolumn{2}{|c|}{ Muestra total } & \multicolumn{2}{c|}{$\begin{array}{c}\text { Hogares con niños menores } \\
\text { de } 18 \text { años }\end{array}$} \\
\cline { 2 - 5 } & Pavo & Pollo & Pavo & Pollo \\
\hline Es una carne sana y saludable & $\mathbf{8 7 , 8 4}$ & 79,36 & $\mathbf{9 1 , 3 5}$ & 75,68 \\
\hline $\begin{array}{l}\text { Se encuentra fácilmente en los } \\
\text { establecimientos donde realizo las compras }\end{array}$ & 67,84 & $\mathbf{9 9 , 5 2}$ & 69,19 & $\mathbf{9 9 , 4 6}$ \\
\hline Tiene una buena relación calidad - precio & 66,08 & $\mathbf{9 3 , 7 6}$ & 68,11 & $\mathbf{9 4 , 0 5}$ \\
\hline Es una carne sabrosa & 55,36 & $\mathbf{8 7 , 2 0}$ & 62,16 & $\mathbf{8 3 , 7 8}$ \\
\hline Es una carne fácil y rápida de cocinar & 79,04 & $\mathbf{9 6 , 3 2}$ & 87,03 & $\mathbf{9 5 , 1 4}$ \\
\hline Es una carne de alta calidad & $\mathbf{7 8 , 8 8}$ & 77,76 & $\mathbf{7 9 , 4 6}$ & 73,51 \\
\hline Se puede preparar de muchas maneras & 71,84 & $\mathbf{9 8 , 7 2}$ & 77,30 & $\mathbf{9 9 , 4 6}$ \\
\hline Es digestiva, no es pesada & 81,44 & $\mathbf{8 3 , 8 4}$ & $\mathbf{8 7 , 0 3}$ & 83,24 \\
\hline $\begin{array}{l}\text { Es una carne con precio atractivo, } \\
\text { económico }\end{array}$ & 58,56 & $\mathbf{9 4 , 5 6}$ & 57,84 & $\mathbf{9 5 , 1 4}$ \\
\hline
\end{tabular}

Las variables con valores más altos aparecen en negrita.

Fuente: Elaboración propia

\section{Conclusiones}

Podemos concluir que existen diferencias entre los hogares con y sin hijos menores de 18 años en los hábitos de consumo y de compra de carne de pollo y pavo. A pesar de tener un posicionamiento similar en la mente de los consumidores, existen diferencias significativas en la frecuencia de consumo y de compra entre ambos tipos de carne (diferenciando entre carne fresca y elaborados).

En cambio, no hay diferencias en la imagen de ambas carnes entre el total de la muestra y los hogares con hijos menores de 18 años. Gracias al análisis de la imagen, vemos que ambas carnes se perciben como sanas y saludables, pero la de pollo se relaciona con ser una carne más arraigada y con usos más prácticos.

\section{Agradecimientos}

Esta investigación ha sido financiada por la Generalitat Valenciana (Valencia, España) con la subvención [AICO/2017/066] (Proyecto "Sostenibilidad de la cadena de valor alimentaria: De la producción al consumo responsable").

\section{Bibliografía}

Baviera-Puig, A., Montero-Vicente, L., Escribá-Pérez, C., y Buitrago-Vera, J. (2021). “Analysis of chicken and turkey meat consumption by segmentation of Spanish consumers using food-related lifestyle". Spanish Journal of Agricultural Research, 19(1), e0101. https://doi.org/10.5424/sjar/2021191-16419

Cea, M. A. (2010). Métodos de Encuesta. Teoría y Práctica, Errores y Mejora. Síntesis, S.A., Madrid.

Cofnas, N. (2019). "Is vegetarianism healthy for children?" Critical Reviews in Food Science and Nutrition, 59(13), 2052-2060. https://doi.org/10.1080/10408398.2018.1437024

Martini, S., Conte, A., y Tagliazucchi, D. (2019). "Comparative peptidomic profile and bioactivities of cooked beef, pork, chicken and turkey meat after in vitro gastro-intestinal digestion". Journal of Proteomics, 208, 103500. https://doi.org/10.1016/j.jprot.2019.103500

Montero-Vicente, L., Escribá-Pérez, C., Baviera-Puig, A., y Buitrago-Vera, J. (2018). "Analysis of the commercial value of rabbit meat based on positioning of the different types of fresh meat". Spanish Journal of Agricultural Research, 16(3), e0110. https://doi.org/10.5424/sjar/2018163-13407

Pereira, P. M. de C. C., y Vicente, A. F. dos R. B. (2013). "Meat nutritional composition and nutritive role in the human diet". Meat Science, 93(3), 586-592. https://doi.org/10.1016/j.meatsci.2012.09.018

Tang, M., Hendricks, A. E., y Krebs, N. F. (2018). "A meat- or dairy-based complementary diet leads to distinct growth patterns in formula-fed infants: a randomized controlled trial". The American Journal of Clinical Nutrition, 107(5), 734-742. https://doi.org/10.1093/ajcn/nqy038 